\title{
Wideband CDMA Modem for Korea Telecom IMT-2000 Testbed System
}

\author{
June Chul Roh*, Yong Ho Kim, Chan Eui Yun*, Hyun Myung Pyo*, and Gilsoon Kang*** \\ * Wireless Communications Research Lab., Korea Telecom \\ 17 Woomyun-dong, Seocho-gu, Seoul, 137-792, Korea \\ Phone: +82-2-526-6158, Fax.: +82-2-526-5568, E-mail: jcroh@kt.co.kr \\ ** Seodu Inchip, Inc., 142-21 Samsung-dong, Kangnam-gu, Seoul, 135-090, Korea
}

\begin{abstract}
This paper describes the design and implementation of wideband code division multiple access (CDMA) modem for the Korea Telecom (KT) International Mobile Telecommunications2000 (IMT-2000) testbed system. We have been working on two air interfaces, one of which is based on inter-cell synchronous CDMA and the other on inter-cell asynchronous CDMA. Both air interfaces are designed to accommodate chip rates of 4.196 , 8.192, and 16.384 Mcps (chip per second) corresponding to radio frequency (RF) bandwidths of 5,10 , and $20 \mathrm{MHz}$, respectively. These two air interfaces are being implemented in the first and second testbed systems, respectively. In this paper, we focus on the first phase system, especially on the design and implementation of wideband CDMA modem.
\end{abstract}

Index Terms--Spread-spectrum, CDMA, IMT-2000, Modem, ASIC

\section{INTRODUCTION}

Most of the second generation digital cellular and personal communication service (PCS) systems operating in these days are designed to provide relatively low rate voice-oriented services. Therefore, in these systems there are difficulties in accommodating emerging wireless communication services that require higher data rates, enhanced quality of service, and various traffic types such as voice, wireless packet data, and wireless multimedia. Standardization process for a new generation mobile radio service, namely IMT-2000, is on its almost final stage under ITU-R. Several radio transmission technologies (RTTs) were proposed and will be evaluated and selected as the IMT-2000 RTT standard.

Wideband CDMA based on direct-sequence spreadspectrum is considered as a key candidate technology satisfying many requirements of the third generation mobile radio systems. A large number of digital cellular and PCS systems are employing CDMA as their multiple access schemes. The chip rate of these systems, which determines the RF bandwidth, is relatively low (1.2288 Mcps). Therefore, it is difficult that the many advantages of spread-spectrum multiple access [1]-[3] are fully gained in these systems. In order to take advantage of the good characteristics of spread-spectrum signals, many of IMT-2000 RTT proposals are employing wideband CDMA techniques [4]-[8]. Another of the reasons for using wideband CDMA is that many services of the IMT2000 require higher date rates. Therefore, if a certain minimum processing gain is maintained, the chip rate increases according to the data rate.

Korea Telecom Wireless Communications Research Laboratory has investigated and implemented IMT-2000 testbed systems employing wideband CDMA based on directsequence spread-spectrum techniques as their air interfaces. We have been working on two air interfaces, one of which is based on inter-cell synchronous CDMA and the other on inter-cell asynchronous CDMA. Both air interfaces are designed to accommodate chip rates of 4.196, 8.192, and 16.384 Mcps corresponding to RF bandwidths of 5, 10, and $20 \mathrm{MHz}$, respectively. The two systems have their own advantages and disadvantages relative to each other. The two internal air interfaces are somewhat different with the Korea IMT-2000 RTT proposals, namely Telecommunication Technology Association (TTA) proposal I [5] and TTA proposal II [6].

The two KT-internal air interfaces are being implemented in the first and second testbed system, respectively. The first phase system uses a chip rate of $8.192 \mathrm{Mcps}$, and supports the wireless services that need a data rate up to $144 \mathrm{kbps}$. The purpose of developing the IMT-2000 testbeds is to evaluate KT air interfaces, and to develop wideband CDMA modem technologies, application services, and wireless ATM network systems.

In this paper, we focus on the first phase system, especially on the design and implementation of wideband CDMA modem. In Section II, we introduce the KT IMT-2000 air interface and testbed system. Design and implementation issues of wideband CDMA modem will be described in Section III, followed by summary and conclusions in Section IV.

\section{KOREA TELECOM IMT-2000 AIR INTERFACE AND TESTBED SYSTEM}

\section{A. Air Interface}

In this section, we briefly describe the air interface for Korea Telecom IMT-2000 testbed system, mainly the first phase 
testbed. The air interfaces of the first and the second phase system are summarized in Table 1.

Table 1. Summary of the air interfaces for Korea Telecom IMT-2000 testbed systems.

\begin{tabular}{|c|c|c|}
\hline Items & Phase 1 & Phase 2 \\
\hline Multiple Access & Wideband DS-CDMA & Wideband DS-CDMA \\
\hline Duplex & FDD & FDD \\
\hline $\begin{array}{l}\text { PN Chip Rate } \\
\text { (Mcps) }\end{array}$ & 1.024/4.096/8.192/16.384 & $1.024 / 4.096 / 8.192 / 16.384$ \\
\hline $\begin{array}{l}\text { Carrier Spacing } \\
(\mathrm{MHz})\end{array}$ & $1.25 / 5 / 10 / 20$ & $1.25 / 5 / 10 / 20$ \\
\hline $\begin{array}{l}\text { Frame Length } \\
(\mathrm{ms})\end{array}$ & 16 & 10 \\
\hline Channel Coding & $\begin{array}{l}\text { Convolutional coding } \\
(\mathrm{R}=1 / 2, \mathrm{~K}=7)\end{array}$ & $\begin{array}{l}\text { Convolutional coding } \\
(\mathrm{R}=1 / 2, \mathrm{~K}=9) \text { \& } \\
\text { opt. Reed-Solomon (RS) } \\
\text { code }\end{array}$ \\
\hline $\begin{array}{l}\text { Channel } \\
\text { Structure }\end{array}$ & $\begin{array}{l}\text { FL: Pilot/Sync/Paging/ } \\
\text { Traffic/Signaling } \\
\text { RL: Pilot/Access/Traffic/ } \\
\text { Signaling }\end{array}$ & $\begin{array}{l}\text { FL: Plot/Sync/Paging/ } \\
\text { Traffic/Signaling } \\
\text { RL: Pilot/Access/Traffic/ } \\
\text { Signaling }\end{array}$ \\
\hline $\begin{array}{l}\text { Spreading } \\
\text { Codes }\end{array}$ & $\begin{array}{l}\text { Short PN code } \\
\text { Long PN Code }\end{array}$ & $\begin{array}{l}\text { Short Gold code } \\
\text { Long PN Code }\end{array}$ \\
\hline Signaling & $\begin{array}{l}\text { Dedicated code channel for } \\
\text { signaling }\end{array}$ & $\begin{array}{l}\text { Dedicated code channel for } \\
\text { signaling }\end{array}$ \\
\hline $\begin{array}{l}\text { Orthogonal } \\
\text { Codes }\end{array}$ & Walsh codes & Walsh codes \\
\hline $\begin{array}{l}\text { Inter-cell } \\
\text { Synchronization }\end{array}$ & Synchronous & Asynchronous \\
\hline $\begin{array}{l}\text { Data mod./ } \\
\text { PN Spreading }\end{array}$ & FL \& RL: QPSK/QPSK & FL \& RL: QPSK/QPSK \\
\hline Detection & $\begin{array}{l}\text { FL \& RL: Pilot Assisted } \\
\text { Coherent Detection }\end{array}$ & $\begin{array}{l}\text { FL \& RL: Pilot Assisted } \\
\text { Coherent Detection }\end{array}$ \\
\hline $\begin{array}{l}\text { Multirate Con- } \\
\text { cept }\end{array}$ & Multi-code & $\begin{array}{l}\text { Multi-code and/or variable } \\
\text { spreading factor }\end{array}$ \\
\hline & & (Note: Subject to change) \\
\hline
\end{tabular}

FL: forward link, $R L$ : reverse link

The air interface is wideband, spread-spectrum radio interface that uses CDMA techniques. In the testbed system, a CDMA system using a chip rate of 8.192 Mcps is implemented, which requires about $10 \mathrm{MHz}$ bandwidth. Frequency division duplex (FDD) is adopted, in which the forward link carrier frequency is $2115 \mathrm{MHz}$ and the reverse link 1890 MHz. The frame length is $16 \mathrm{~ms}$, which corresponds to the period of the pilot PN codes in time.

The forward link is composed of Pilot, Sync, Paging, Traffic, and Signaling channels. Orthogonality among the forward link channels originated from a base station (BS) is maintained by using orthogonal Walsh functions. Data bit stream of each channel except the Pilot channel is encoded by convolutional code with half rate and constraint length of 7 , repeated to match a basic symbol rate of $64 \mathrm{ksps}$ (symbol per second), and then interleaved, and QPSK modulated. The resulting symbol streams on inphase (I) and quadrature (Q) channel are spread by I and Q pilot pseudo-noise (PN) sequence with period of $2^{17}$, respectively. Each BS in the system is identified by the time offset of the pilot PN codes. The forward link structure is similar to that of IS-95 except in some differences such as the chip rate, the period of pilot PN codes, and assignment of dedicated code channel for signaling.

In the reverse link, we adopt continuous reverse pilot channel from each mobile station (MS) to base station (BS). Using continuous pilot in the reverse link makes demodulation of reverse link channels at the BS easier, and therefore the hardware complexity of the BS modem can be reduced. Because all MSs in the system transmit their own pilot channels that are not synchronized with each other, multiple access interference (MAI) at the demodulator of the BS increases. The pilotto-traffic power ratio of the reverse link mainly determines the MAI and the performance gain from coherent demodulation. It has been shown from many recent efforts to optimize the power ratio that the pilot power should be less than that of the traffic channel, say, by $6 \mathrm{~dB}$, and that using pilot channels in the reverse link in an optimized way gives better performance than noncoherent case.

The reverse link consists of Pilot, Access, Traffic, and Signaling channel. The orthogonality among the reverse link channels from an MS is maintained by using the orthogonal Walsh functions, and the reverse link channels from different MSs are addressed by long codes with different time offsets.

To support data rates greater than $64 \mathrm{kbps}$, multi-code scheme is applied to the both links. For example, to support a service requiring $128 \mathrm{kbps}$ of data rate, traffic data are transmitted on two different $64 \mathrm{kbps}$ orthogonal code channels in parallel. We are considering orthogonal variable spreading factor (OVSF) codes [4], [7] to support multi-rate data services.

\section{B. Testbed System}

In the first phase testbed system, we have designed and im-

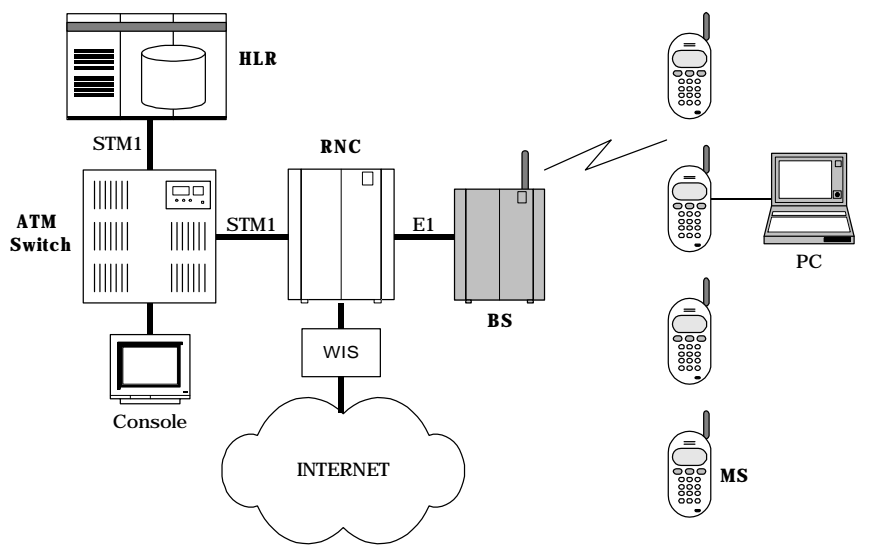

Fig. 1. Overall system architecture of the KT IMT-2000 testbed system.

plemented an inter-cell synchronous CDMA system that has a chip rate of $8.192 \mathrm{Mcps}$, and that supports wireless communication services of up to $144 \mathrm{kbps}$. The services to be tested include $32 \mathrm{kbps}$ ADPCM voice telephone service, $128 \mathrm{kbps}$ video telecommunication service, wireless Internet access, and user identification module (UIM) application services.

The testbed system is configured with a BS, a radio network controller (RNC), and an ATM switch. The BS has an RF transceiver, an analog interface module, clock and reference signal generation card, and channel cards. Each channel card has wideband CDMA modem ASICs and performs baseband signal processing and control functions related with the ASICs. 


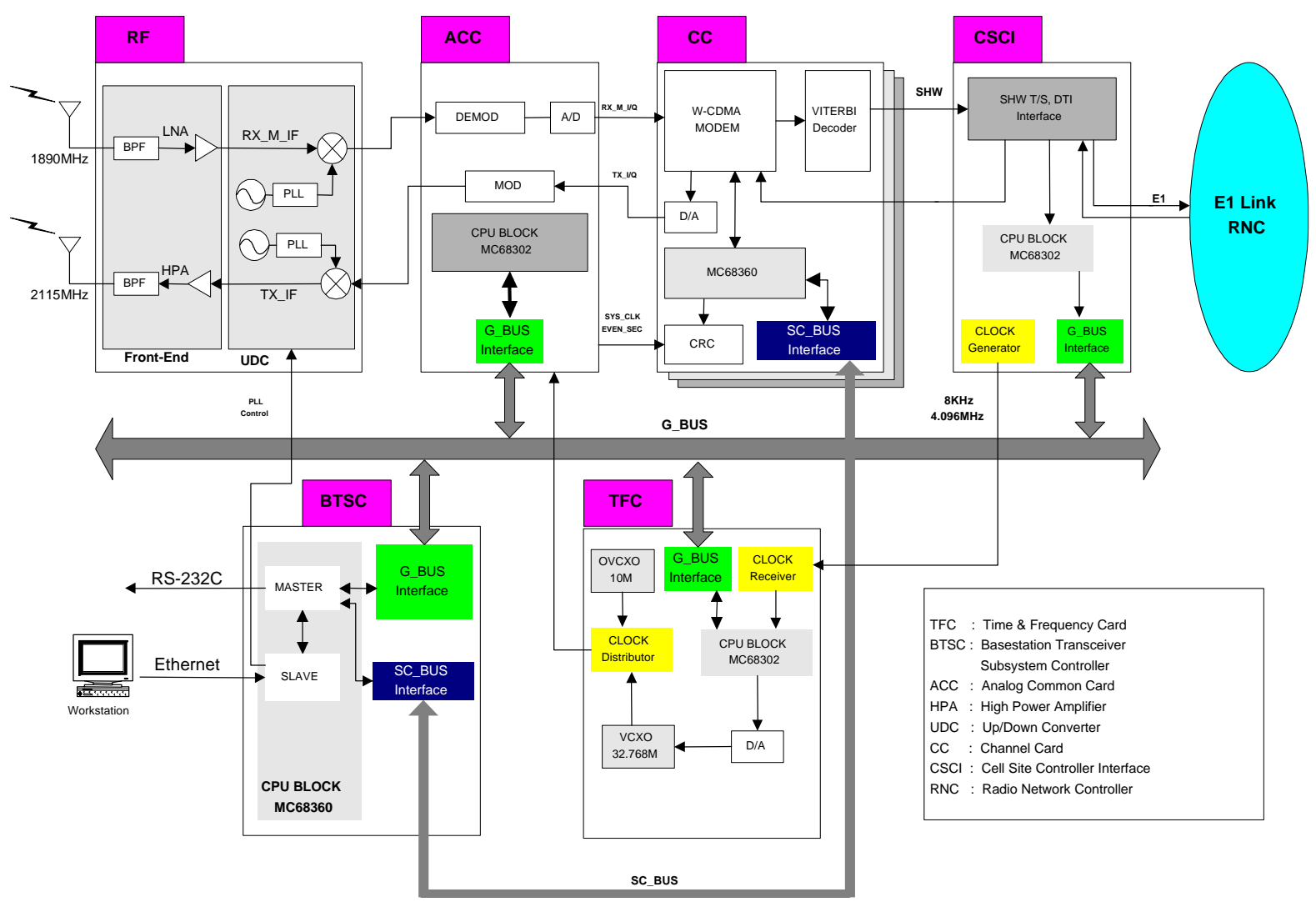

Fig. 2. Board-level functional blocks of the base station.

The channel card is designed to support two base station modem ASICs which can be controlled independently. Each modem ASIC can support three orthogonal code channels, which can be configured to support data rates of $8,16,32$, or 64 kbps, independently. Fig. 1 and 2 shows the overall system architecture of the KT IMT-2000 testbed system and the board-level functional blocks of the BS, respectively.

\section{WIDEBAND CDMA MODEM DESIGN AND IMPLEMENTATION}

Transmitting wideband direct-sequence spread-spectrum signals and receiving them reliably in a cellular environment which has multipath fading characteristics are very challenging in implementing wireless multimedia communication system. In our system, most of the signal processing necessary in modulation and demodulation of wideband CDMA signals are implemented in the BS modem ASIC and the MS modem ASIC. In this section, we describe the design and implementation of wideband CDMA modem ASICs for the KT IMT2000 testbed system.

One BS modem ASIC has three transmit and receive channels. Each transmit channel can be configured as the pilot, the control, the user traffic, or the signaling channel, and each receive channel can be configured as the user traffic or sig-

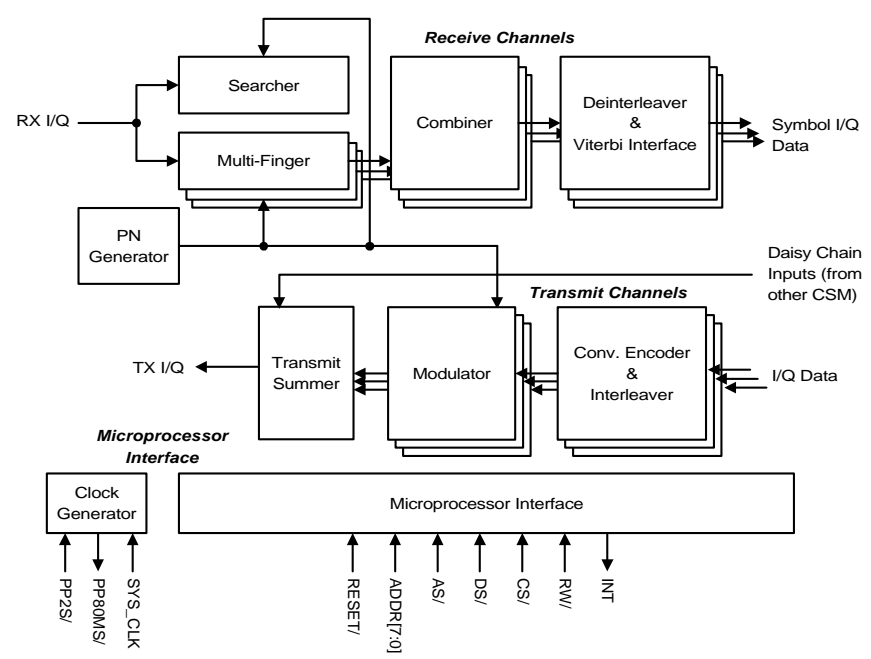

Fig. 3. Top-level block diagram of the BS modem ASIC.

naling channel, independently. And, one MS modem ASIC can support three traffic and/or signaling channels. Therefore, one MS that can have up to three code channels, i.e., two traffic channels and one signaling channel, is supported with one BS modem ASIC and one MS modem ASIC. Fig. 3 is the toplevel block diagram of the BS modem ASIC. 
In order to lessen the CPU processing load, each channel interfaces to CPU or E1 according to its characteristic. The control and the signaling channel interfaces with CPU, and the user traffic channel with E1 because no signaling information is in the user traffic channel, which is one of the benefits that can be obtained by using dedicated code channel for signaling.

The transmit section of the wideband CDMA modem ASIC has the functional blocks for convolutional encoding, symbol repetition, block interleaving, spreading by pilot $\mathrm{PN}$ sequence, pulse-shaping filtering, and transmit channel combining. Fig. 4 shows the transmit section of the BS modem ASIC. The transmit data from CPU or E1 bus is written to the input buffer. The data is encoded, repeated, interleaved, multiplied by decimated long code, multiplied by Walsh sequence, and spread by I and Q pilot PN codes. The resulting chip symbol stream is filtered by finite impulse response (FIR) pulseshaping filters to form the transmit signal. An equi-ripple FIR filter with 48 taps is used as the pulse-shaping filter. The transmit signals from the three transmit channels are summed up in the channel summer. The modem ASIC has a daisychain summer that adds the transmit signals from its channel summer and the output signal of other modem ASIC in order to add the transmit output signals from the modem ASICs in a channel card in digital domain.

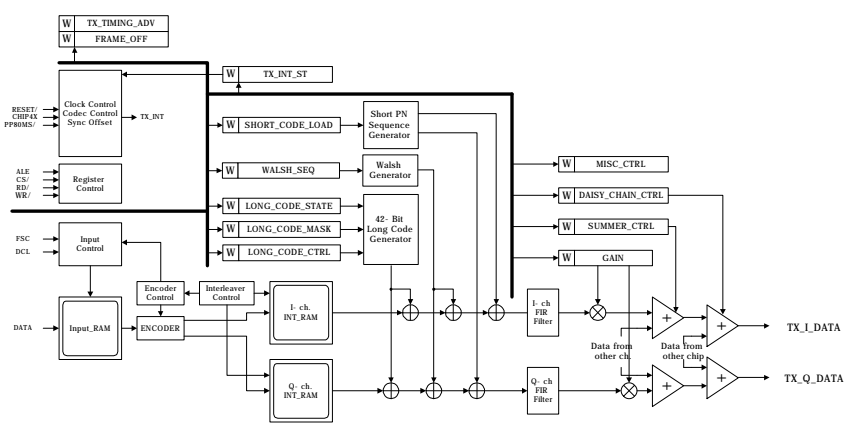

Fig. 4. Transmit section of the BS modem ASIC.

The receive section of the modem requires many complicated signal processing and/or control functions to demodulate incoming wideband CDMA signals. First, the modem has to search the PN offset of the incoming receive signals to acquire local PN signals synchronized to the received signal, and from this the system time is also acquired. As acquisition scheme [2], [3], we use the double-dwell serial search method in the MS modem and single-dwell serial search method in the BS modem. The searcher in the modem ASIC does such initial time synchronization. The searcher searches a defined PN hypothesis window and reports the search results such as energies and their PN positions to CPU. The searcher operation can be controlled with the related registers that determine parameters such as the number of the PN hypotheses, the coherent integration time, and the threshold values. Fig. 5 is the searcher of the BS modem ASIC.

The PN code phases determined by the searcher are assigned to the multi-finger which has the functions of symbol demodulation, PN code time-tracking, and lock detection. Fig.

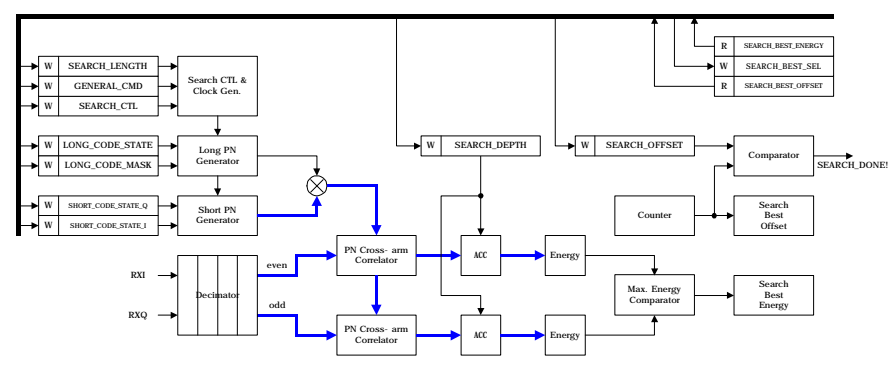

Fig. 5. Searcher of the BS modem ASIC.

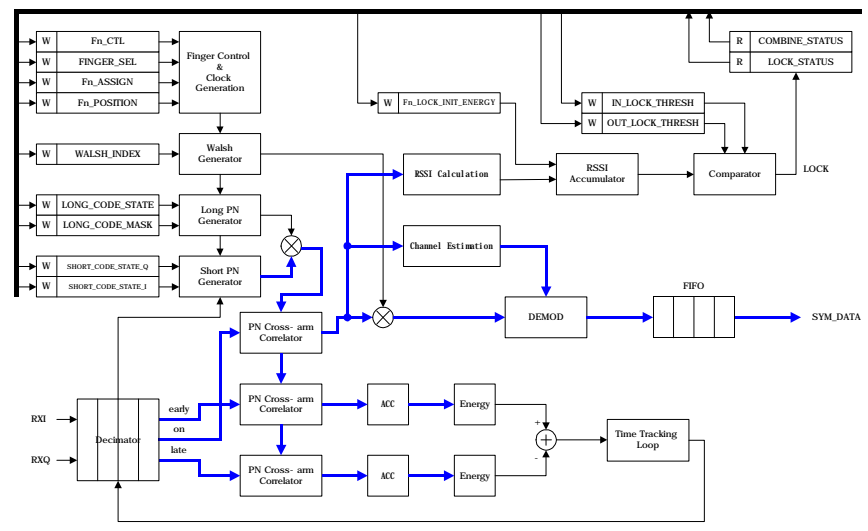

Fig. 6. Finger of the BS modem ASIC.

6 shows the finger of the BS modem ASIC. The finger uses three samples that the decimator outputs, i.e., on-time sample for demodulation, and early and late samples for timetracking. A delay-locked tracking loop [2], [3] is implemented for time-tracking. The loop bandwidth of the time-tracking loop can be controlled with a register.

For demodulation, on-time samples are despread by locally generated spreading codes and Walsh code, and then coherently demodulated making use of the pilot signal. The demodulated output symbols from multi-finger are combined with maximal ratio combining method. The demodulated symbols after the combiner are adapted to the bit width of the Viterbi decoder. We use a commercial Viterbi decoder chipset in our testbed system.

The forward pilot signal is also used in the automatic frequency control (AFC) block of the MS modem ASIC. The AFC loop estimates the frequency error from the unmodulated pilot carrier [10], [11] and outputs this error to the analog interface with pulse density modulation (PDM) format. An automatic gain control (AGC) loop and a DC offset compensation block are also implemented in the modem ASIC. Other miscellaneous blocks for CPU interface, code generation, and reference time generation are also provided in the ASIC.

In developing the wideband CDMA modem ASICs, we started by designing the transmitter and receiver algorithms and simulating the algorithms by using C-programming language. Also we have performed integer simulations to select the bit-width for each data path. With the integer simulation results, we designed the ASIC architecture considering the 
control functions, and then defined the register set and related operations. A VHDL-based logic synthesis methodology is used in the gate-level ASIC design.

Fig. 7 shows the waveform and its spectrum of the transmitted pilot signal after digital-to-analog (DA) conversion. Demodulated symbols from the MS modem ASIC and decoded data from the Viterbi decoder are shown in Fig. 8.

\section{SUMMARY AND CONCLUSIONS}

In this paper, the KT wideband CDMA air interface and testbed system were explained. And, we described the design and implementation issues of wideband CDMA modem for the testbed system.

An inter-cell synchronous CDMA system of a chip rate of 8.192 Mcps has been implemented in the testbed system. The wideband CDMA modem ASICs for BS and MS have been developed for the signal processing in modulation and demodulation of wideband CDMA signals. Power control and hand over are not realized in this system, but we are considering them in the next testbed system.

\section{ACKNOWLEDGEMENT}

The authors acknowledge Hanwha/Telecom Co. for the cooperation in developing the KT IMT-2000 testbed system and Mr. Byung Yang Lee for his helpful advice in regard to the RF interface.

\section{REFERENCES}

[1] R. L. Pickholtz, D. L. Schilling, and L. B. Milstein, "Theory of spread-spectrum communications - a tutorial," IEEE Trans. Commun., vol. COM-30, pp. 855-884, May 1982.

[2] M. K. Simon, J. K. Omura, R. A. Scholtz and B. K. Levitt, Spread Spectrum Communications Handbook, McGraw-Hill, New York, 1994.

[3] A. J. Viterbi, CDMA: Principles of Spread Spectrum Communication, Addison-Wesley, 1995.

[4] Japan's Proposal for Candidate Radio Transmission Technology on IMT-2000: W-CDMA, Association of Radio Industries and Businesses (ARIB), June, 1998.

[5] Global CDMA I: Multiband Direct-Sequence CDMA System RTT System Description Ver. 1.0, Telecommunication Technology Association (TTA), June 1998

[6] Global CDMA II for IMT-2000 RTT System Description Ver. 1.0, TTA, June 1998.

[7] The ETSI UMTS Terrestrial Radio Access (UTRA) ITU-R RTT Candidate Submission, European Telecommunications Standards Institute (ETSI), July 1998.

[8] The cdma2000 ITU-R RTT Candidate Submission, TIA TR45.5, June 1998.

[9] Yong Ho Kim, June Chul Roh, Hyun Myung Pyo, and Myung Sang Yoon, "Korea Telecom IMT-2000 testbed based on wideband CDMA technologies," ACTS Mobile Commun. Summit '98, June 8-11, 1998, Rhodes, Greece, pp. 691-696.

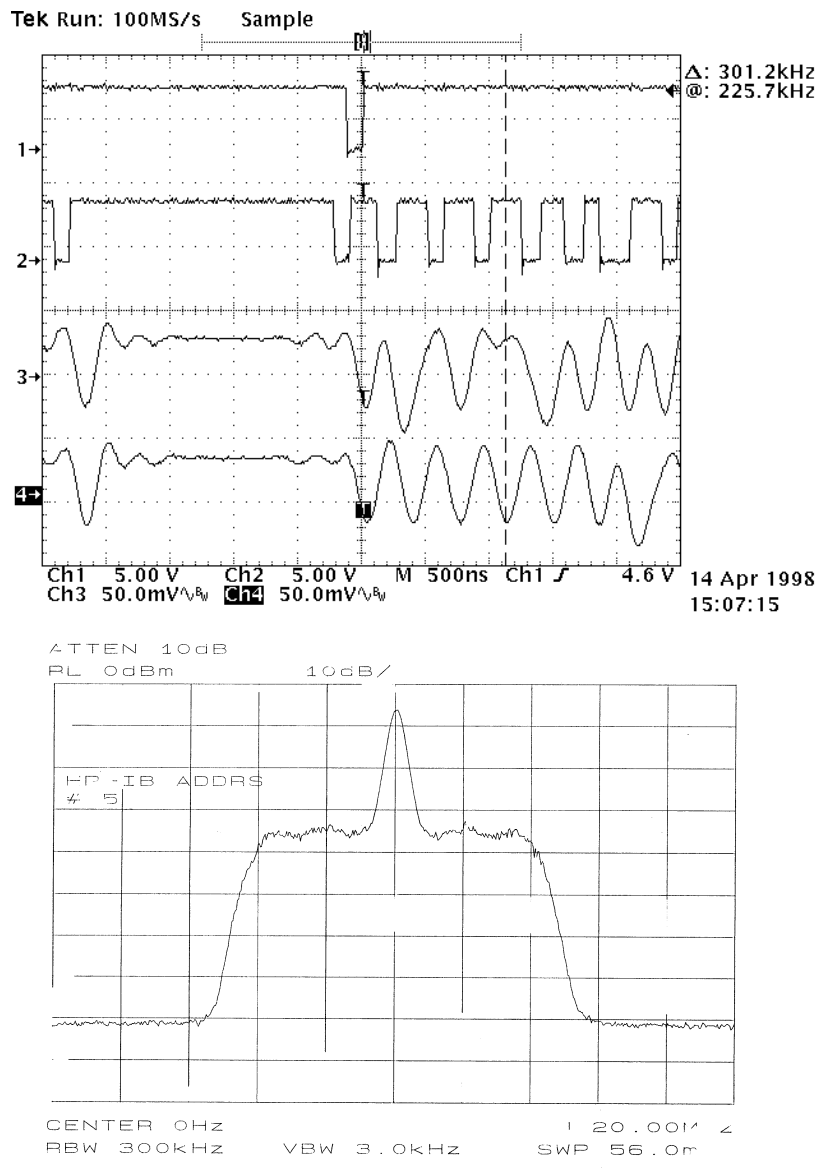

Fig. 7. Waveform and spectrum of the pilot signal after DA conversion.

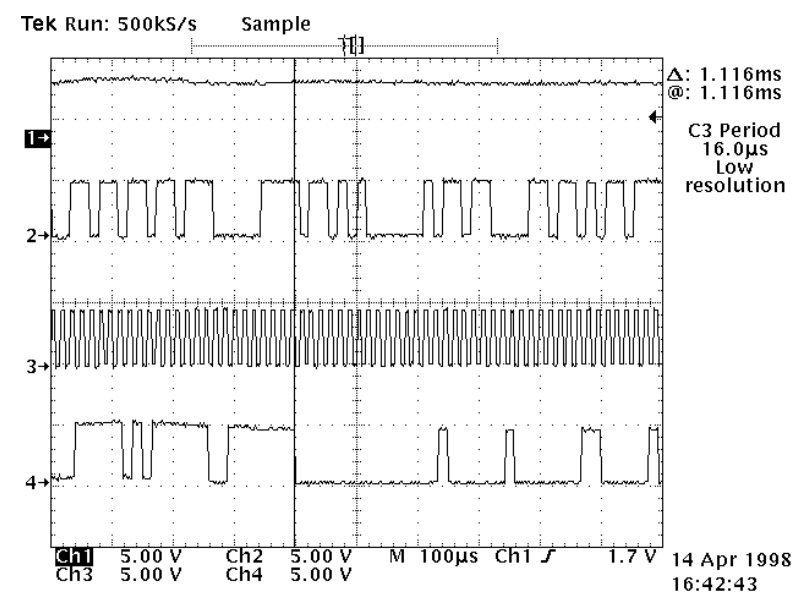

Fig. 8. Demodulated Symbols (waveform 2) from the MS modem ASIC and decoded data (waveform 4) from the Viterbi decoder. Only MSBs of the symbols are shown. Data of 0x00, 0x01, 0x02, ... were transmitted at $8 \mathrm{kbps}$. The solid vertical line is the frame boundary. 